Bull. Austral. Math. Soc.

$54 \mathrm{~F} 05,06 \mathrm{~F} 30,54 \mathrm{D} 65,54 \mathrm{D} 99$

VOL. 55 (1997) [131-142]

\title{
TOPOLOGICAL SEPARABILITY AND AXIOMS OF COUNTABILITY IN GPO-SPACES
}

\author{
J.C.R. Alcantud
}

\begin{abstract}
In this paper we obtain characterisations of first and second countability and separability for GPO-spaces, a class of topological spaces that include LOTS and GO-spaces. Some additional results concerning the transmission of these properties to weaker/finer topologies are derived in this framework.
\end{abstract}

\section{INTRODUCTION}

A LOTS (for "Linearly Ordered Topological Space") is a triple $\left(X,<, \tau_{o r}(<)\right)$, where $(X,<)$ is a linearly ordered set and $\tau_{o r}(<)$ denotes the usual order topology obtained from <. If $Y$ is a subset of $X$, it may happen that the subspace topology $\left.\tau_{o r}(<)\right|_{Y}$ is strictly larger than the order topology associated with the restricted linear order $<\left.\right|_{Y}$. Subspaces of LOTS are called GO-spaces (for "Generalised Ordered Space"), and have been studied, for example, by Cech [2], Faber [5], Heath, Lutzer and Zenor [7] and Lutzer [8]. Besides their intrinsic interest, we mention that they have provided many pathological examples in general topology: the Sorgenfrey line, the Michael line (see Michael [10]), and the example of a Michael Space constructed under the Continuum Hypothesis by this author in [11] are GO-spaces.

A more general type of order than a linear order is a preference (asymmetric, negatively transitive). The latter have applications in a number of fields, which include mathematical economics (see Fishburn [6], Debreu [3] and [4] among others). The author and Gutiérrez introduced in [1] generalisations of both LOTS and GO-spaces for spaces ordered by preferences, namely POTS (for "Preference-Ordered Topological Space") and GPO-spaces (for "Generalised Preference-Ordered Space") respectively. Such topological spaces were shown to exhibit properties similar to those of their linear analogues. In particular, GPO-spaces are monotonically and hereditarily normal, and completely regular. The technique that was used to obtain such results produces characterisations of many other topological properties through the study of certain associated GO-spaces.

Received 28th February, 1996

The author is grateful to J. M. Gutiérrez for the considerable help he has extended. Most of this work was done at the Department of Mathematics of the London School of Economics. This research was supported by DGICYT Project PB94-1385.

Copyright Clearance Centre, Inc. Serial-fee code: 0004-9729/97 \$A2.00+0.00. 
This paper is concerned with the study of first and second countability and separability in GPO-spaces, which constitute a more general framework than that of GOspaces. Every separable GPO-space is first countable (see Alcantud and Gutiérrez [1]). Separability and second countability may be characterised in terms of separation properties of the order for POTS (see Mehta [9]), but we have not found a similar result for first countability in the literature. In this work the three properties considered will be characterised for GPO-spaces. Some additional results are derived. Given a space ordered by a preference, and considering the topologies on it that produce a GPO-space, then it is shown that first countability is preserved when the topology is strengthened, and second countability is preserved when it is weakened.

\section{Definitions AND REVIEW OF RESUlTS}

Unless otherwise specified, in this section we follow the terminology of Wilansky [12].

We recall some definitions. An asymmetric, negatively transitive binary relation < on a set is a preference. If a preference $<$ satisfies that either $x<y$ or $y<x$ for two different elements $x$ and $y$, then $<$ is called a linear (or total) order. We say that $X$ is a set ordered by a preference $<$ and $X$ is linearly ordered by < respectively. We may associate with a preference $<$ an equivalence relation called the indifference relation; it is denoted by $\sim$ and defined by $x \sim y$ if and only if not $x<y$, not $y<x$. By $x \preceq y$ we shall mean $x<y$ or $x \sim y$; this relation is complete and transitive. If $<$ is a linear order we shall use the more explicit notation $x \leqslant y$ if and only if $x<y$ or $x=y$, since the indifference is trivial in this case. Therefore the relation $\leqslant$ is antisymmetric for any linear order $<$.

The quotient set by the indifference $\sim$ will be denoted by $X / \sim$. Its elements (equivalence relations) will be denoted by $[x]_{\sim}$.

Two immediate properties of preferences that we shall make use of are (see Fishburn [6, Theorem 2.1]:

(i) for every $a, x, y, z \in X: a \sim y<z$ implies $a<z$ and $x<y \sim a$ implies $x<a$

(ii) for every $x, y \in X$ : exactly one of $x<y, x \sim y$ or $y<x$ is true.

A transitive, asymmetric (or irreflexive) binary relation $<$ is called a partial order. Preferences are examples of partial orders. The order topology associated with a partial order $<$ (denoted by $\tau_{o r}(<)$, or simply $\tau_{o r}$ if no confusion is possible) is the topology which has the family formed by $X$ and all the subsets

$$
\{a \in X: a<x\} \text { and }\{a \in X: x<a\}
$$

with $x \in X$ as a subbase of open sets. 
For a partially ordered space $(X,<)$ we adopt the following convention: given $A \subseteq$ $X$ and $x \in X, x<A(A<x)$ if and only if $x<a(a<x)$ for all $a \in A$; consequently $x<\emptyset$ (and $\emptyset<x$ ). We denote $A^{l}=\{x \in X: x<A\}, A^{u}=\{x \in X: A<x\}$; consequently $\emptyset^{l}=\emptyset^{u}=X$. The base $\mathcal{B}$ of $\tau_{o r}(<)$ formed by finite intersections of elements from the defining subbase above is

$$
\mathcal{B}=\{\{x \in X: S<x<T\} \text { with } S, T \subseteq X \text { finite }\}=\left\{S^{u} \cap T^{l} \text { with } S, T \subseteq X \text { finite }\right\}
$$

Notice that $T=\emptyset$ and $S=\emptyset$ account for the cases when all the elements intersected are of the type $\{x \in X: a<x\}$ or the type $\{x \in X: x<b\}$.

The following convention was introduced in Alcantud and Gutiérrez [1] in order to obtain a simple expression for $\mathcal{B}$ in the preference case. When we refer to the order topology derived from a preference the use of the symbols $+\infty$ and $-\infty$ will be defined by: each $x \in X$ satisfies $x<+\infty$ and $-\infty<x$. The base $\mathcal{B}$ of $\tau_{\text {or }}(<)$ will be expressed simply by

$$
\mathcal{B}=\{\{x \in X: a<x<b\} \text { with } a, b \in X \cup\{ \pm \infty\}\} .
$$

We now proceed to define the topological spaces to be studied here. Firstly we recall that a subset $C$ of a space ordered by a preference $(X,<)$ is convex if

$$
p \preceq z \preceq q \text { and } p, q \in C \text { imply } z \in C .
$$

For linear orders, this definition may be restated as follows:

$$
p \leqslant z \leqslant q \text { and } p, q \in C \text { imply } z \in C .
$$

Then the following definitions were introduced in Alcantud and Gutiérrez [1]:

Definition 1: A GPO-space (for "Generalised Preference-Ordered space") is a triple $(X,<, \tau)$ where $<$ is a preference on $X$ and $\tau$ is a topology on $X$ such that $\tau_{o r} \leqslant \tau$ and $\tau$ has a base formed by convex sets. A POTS (for "Preference-Ordered Topological Space") is a triple $\left(X,<, \tau_{o r}(<)\right)$ where $<$ is a preference on $X$.

If $<$ is a linear order we obtain a GO-space and a LOTS respectively (as defined, for example, in Faber [5]).

The following result also appeared in Alcantud and Gutiérrez [1], and generalises the classical result of Cech [2] (see Lutzer [8] Theorem 2.9) that the class of GO-spaces coincides with the class of subspaces of LOTS:

Proposition 1. The class of GPO-spaces and the class of subspaces of POTS coincide.

Furthermore, in [1] we provided a useful characterisation of GPO-spaces in terms of bases: 
Proposition 2. Let $X$ be a set ordered by a preference < and $\tau$ a topology on $X$. Then $(X,<, \tau)$ is a GPO-space if and only if $\tau$ has a base consisting of all the sets

$$
\{x \in X: a<x<b\} \text { with } a, b \in X \cup\{ \pm \infty\}
$$

and possibly some sets of any of the following three types:

$$
\begin{aligned}
& \{x \in X: a<x \preceq b\} \text { with } a \in X \cup\{-\infty\} \text { and } b \in X \\
& \{x \in X: a \preceq x<b\} \text { with } b \in X \cup\{+\infty\} \text { and } a \in X \\
& \{x \in X: a \preceq x \preceq b\} \text { with } a, b \in X .
\end{aligned}
$$

\section{ChaRACTERISATIONS OF SEPARABILITY AND COUNTABILITY AXIOMS}

We begin by characterising separability in GPO-spaces. The following concept of order-separability was used in Mehta [9] to characterise topological separability in POTS:

Definition 2: A set $X$ ordered by a partial order $<$ is weakly separable if there exists a countable set $Z \subseteq X$ such that if $x, y \in X, x<y$ and $\{a \in X: x<a<y\} \neq \emptyset$, then there exists some $z \in Z$ such that $x<z<y$.

In the above mentioned paper, Mehta showed that topological separability and weak separability of the preference are equivalent in any partially ordered set endowed with the order topology; particularly, in any POTS. This result may be generalised to consider GPO-spaces, in the following way:

Proposition 3. Let $(X,<, \tau)$ be a GPO-space. Then, $\tau$ is separable if and only if $<$ is weakly separable and $\left\{[x]_{\sim} \in X / \sim:[x]_{\sim} \in \tau\right\}$ is countable.

Proof: We first prove the necessity of the condition. Since $\tau_{o r}(<)$ is obviously separable, Mehta's characterisation implies that $<$ is weakly separable. Furthermore, there are at most countably many open equivalence classes: given a countable dense set $D$ for $\tau$, every two different open classes contain different elements of $D$.

Conversely, let us take a countable subset $Z$ of $X$ given by the definition of weak separability. Let us take a further countable subset $A$ of $X$ that contains some element of each open equivalence class and the maximum and minimum elements of $X$ (if such exist). Define the countable subset $D:=Z \cup A$ of $X$. We shall show that $D$ is dense in $\tau$ : for every $U \in \tau$ we shall find an element of $D$ contained in $U$. Indeed, if $U \in \tau$ then there exists an open set $B$ from a base given by Proposition 2 that satisfies $B \subseteq U$, and two cases arise:

CASE 1. Suppose that we may select such a $B$ of the type $\{y \in X: a<y<b\}$ with $a, b \in X \cup\{ \pm \infty\}$. If $a=-\infty$ : either there exists the minimum of $X$ and therefore it 
is in $B \cap D$, or we may assume without loss of generality that $a \in X$ by choosing any $a<b$. It is similar if $b=+\infty$. Therefore, we may restrict the argument to the case $a, b \in X$; but in this case there exists $z \in Z \subseteq D$ such that $z \in B \subseteq U$.

CASE 2. Suppose that no such $B$ can be selected. Then we show that $B$ contains an open equivalence class:

- If $B=\{y \in X: a \preceq y<b\}$, since $\{y \in X: a<y<b\}=\emptyset$ by the negation of Case 1, we obtain $B=[a]_{\sim} \in T$.

- Similarly, if $B=\{y \in X: a<y \preceq b\}$ then $B=[b]_{\sim} \in \tau$.

- If $B=\{y \in X: a \preceq y \preceq b\}$, the subset $B^{\prime}:=\{y \in X: a<y \preceq b\}$ is open because

$$
B^{\prime}=\{y \in X: a \preceq y \preceq b\} \cap\{y \in X: a<y\}=B \cap\{y \in X: a<y\} .
$$

It follows that $B^{\prime}=[b]_{\sim}$ because $\{y \in X: a<y<b\}=\emptyset$, and $B^{\prime} \subseteq B \subseteq U$.

Therefore there exists $a \in D$ belonging to such an open class and then $a \in B \subseteq U$. This completes the proof.

The following further concept of order-separability was used in Mehta [9] to characterise second countability in POTS:

Definition 3: A set $X$ ordered by a preference $<$ is called perfectly separable if it contains a countable subset $Z$ such that if $x, y \in X$ and $x<y$ then there exists $z \in Z$ such that $x \preceq z \preceq y$.

In [9], Mehta showed that second countability of the topology and perfect separability of the preference are equivalent in any POTS, and therefore that perfect separability implies weak separability for any preference. We now proceed to generalise the former result to GPO-spaces:

Proposition 4. Let $(X,<, \tau)$ be a GPO-space. Then $\tau$ is second countable if and only if $<$ is perfectly separable and the set

$$
T:=\left\{[x]_{\sim} \in X / \sim:\{y \in X: x \preceq y\} \in \tau \text { or }\{y \in X: y \preceq x\} \in \tau\right\}
$$

is countable.

Proof: We first prove the sufficiency of the condition. Let $D$ be a countable subset of $X$ obtained by selecting a member of each class belonging to $T$; thus $\{y \in X: x \preceq y\} \in \tau$ or $\{y \in X: y \preceq x\} \in \tau$ imply that there exists $d \in D$ such that $d \sim x$. Let $Z$ be a countable subset obtained from the perfect separability of $<$, and assume that $Z$ contains the following elements (if such exist): the minimum of $X$, the minimum of $X$ without that minimum, the maximum of $X$ and the maximum of 
$X$ without that maximum. Define $Z^{\prime}:=Z \cup D$; let $\mathcal{B}$ be the union of the following countable collections of open sets:

$$
\left\{y \in X: z_{i}<y<z_{j}\right\} \text { with } z_{i}, z_{j} \in Z^{\prime}
$$

all the $\left\{y \in X: z_{i} \preceq y<z_{j}\right\}$ that are open in $\tau$ with $z_{i}, z_{j} \in Z^{\prime}$

all the $\left\{y \in X: z_{i}<y \preceq z_{j}\right\}$ that are open in $\tau$ with $z_{i}, z_{j} \in Z^{\prime}$

all the $\left\{y \in X: z_{i} \preceq y \preceq z_{j}\right\}$ that are open in $\tau$ with $z_{i}, z_{j} \in Z^{\prime}$.

We shall prove that $\mathcal{B}$ is a base of $\tau$. For any $x^{\prime} \in U \in \tau$ there exists an open set $B$ of a base as obtained by Proposition 2 that satisfies $x^{\prime} \in B \subseteq U$. We shall find a $V \in \mathcal{B}$ such that $x^{\prime} \in V \subseteq B \subseteq U$. Without loss of generality we assume that $X$ is non-finite.

CASE 1. Suppose that we can select $B=\{y \in X: a<y<b\}$ with $a, b \in X \cup\{ \pm \infty\}$. Then we may further assume $a, b \in X$. If $a=-\infty$, either there exists $a^{\prime} \in X$ such that $a^{\prime}<x^{\prime}$ and thus we may redefine $a:=a^{\prime} \in X$, or $x^{\prime}$ is the minimum. In this case $z:=x^{\prime} \in Z, B=\{y \in X: z \preceq y<b\}$, and we may assume $b \in X$ ( $X$ is not finite). Now, if there exists $c \in X$ such that $x^{\prime}<c<b$ then there exists $z^{\prime} \in Z$ such that $x^{\prime}<z^{\prime}$, otherwise we have $z^{\prime}:=b \in Z$ because this element is the minimum of $X$ without the minimum; in any case we reach the conclusion since $V:=\left\{y \in X: z \preceq y<z^{\prime}\right\} \in \mathcal{B}$ satisfies the requirement. A similar argument may be used if $b=+\infty$.

By hypothesis there exist $z_{i}, z_{j} \in Z$ with $a \preceq z_{i} \preceq x^{\prime} \preceq z_{j} \preceq b$. Notice that whenever we find $z_{i}, z_{j} \in Z$ such that $a \preceq z_{i}<x^{\prime}<z_{j} \preceq b$ we can establish the claim by arguing that $V:=\left\{y \in X: z_{i}<y<z_{j}\right\}$ satisfies $x \in V \subseteq B \subseteq U$.

Suppose firstly that neither $\left\{y \in X: a<y<z_{i}\right\}$ nor $\left\{y \in X: z_{j}<y<b\right\}$ is empty. Then we may establish the claim as above since there exist $c, d \in X$ such that $a<c<z_{i}, z_{j}<d<b$, and therefore there exist $z_{k}, z_{l} \in Z$ such that $a \preceq z_{k} \preceq c<x^{\prime}<d \preceq z_{l} \preceq b$.

Suppose, on the contrary, that $\left\{y \in X: a<y<z_{i}\right\}=\emptyset$. According to the condition $z_{i} \preceq \boldsymbol{x}^{\prime} \preceq z_{j}$ we have the following cases:

(1.a) If $x^{\prime}<z_{j}$ then $V:=\left\{y \in X: z_{i} \preceq y<z_{j}\right\}$ satisfies the requirement, because $V=\left\{y \in X: a<y<z_{j}\right\} \in \tau$ and thus $V \in \mathcal{B}$.

(1.b) If $x^{\prime} \sim z_{j}$ then two cases arise:

(1.b.i) If $z_{i}<x^{\prime} \sim z_{j}$ then $z_{j}<b$ because $x^{\prime}<b$. If there exists $c \in X$ such that $z_{j}<c<b$, then there exists $z_{k} \in Z$ such that $z_{j}<c \preceq z_{k} \preceq b$, and $V:=\left\{y \in X: z_{i}<y<z_{k}\right\} \in \tau$ satisfies the requirement since $x^{\prime} \in$ $V \in \mathcal{B}$. Otherwise we may take $V:=\left\{y \in X: z_{i}<y \preceq z_{j}\right\}$, because $V=\left\{y \in X: z_{i}<y<b\right\} \in \tau$ and thus $V \in \mathcal{B}$. 
(1.b.ii) If $z_{i} \sim x^{\prime} \sim z_{j}$ then we have $x^{\prime} \in\left[z_{i}\right]_{\sim}=\left\{y \in X: z_{i} \preceq y \preceq z_{j}\right\}$ and also $\left[z_{i}\right]_{\sim}=\left\{y \in X: a<y \preceq z_{j}\right\}$. If there exists $c \in X$ such that $z_{j}<c<b$ then there exists $z_{k} \in Z$ such that $z_{j}<c \preceq z_{k} \preceq b$, and $V:=\left\{y \in X: z_{i} \preceq y<z_{k}\right\}$ satisfies the condition because $V=$ $\left\{y \in X: a<y<z_{k}\right\} \in \tau$ and thus $V \in \mathcal{B}$. Otherwise we may take $V:=\left\{y \in X: a<y \preceq z_{j}\right\}$, because $V=\{y \in X: a<y<b\} \in \tau$ and thus $V \in \mathcal{B}$.

CASE 2. Suppose now $B=\{y \in X: a \preceq y \preceq b\}$. If $a<x^{\prime}<b$ we reason as in Case 1 with $B^{\prime}=\{y \in X: a<y<b\}$. Therefore we may assume without loss of generality that $a \sim x^{\prime}$ (the case $x^{\prime} \sim b$ is similar); thus $B=\left\{y \in X: x^{\prime} \preceq y \preceq b\right\} \in \tau$. The following cases arise:

(2.a) If furthermore $x^{\prime} \sim b$ then $B=\left\{y \in X: y \sim x^{\prime}\right\} \in \tau$. Therefore

$\left\{y \in X: x^{\prime} \preceq y\right\}=\left\{y \in X: y \sim x^{\prime}\right\} \cup\left\{y \in X: x^{\prime}<y\right\} \in \tau$

and (by definition of $D$ ) we have $x^{\prime} \sim z$ for some $z \in Z^{\prime}$. The open set $V:=B$ satisfies the requirement since $B=\{y \in X: z \preceq y \preceq z\} \in \mathcal{B}$.

(2.b) Suppose, on the contrary, that $x^{\prime}<b$. The following cases arise:

(2.b.i) If there is no $x$ such that $x^{\prime}<x<b$ then

$$
\left\{y \in X: x^{\prime} \sim y\right\}=\left\{y \in X: x^{\prime} \preceq y<b\right\} ;
$$

therefore

$\left\{y \in X: x^{\prime} \sim y\right\}=\left\{y \in X: x^{\prime} \preceq y \preceq b\right\} \cap\{y \in X: y<b\} \in \tau$

and thus $V:=\left\{y \in X: x^{\prime} \sim y\right\}$ satisfies the requirement (as above).

(2.b.ii) If there exists $x$ such that $x^{\prime}<x<b$ then $\left\{y \in X: x^{\prime} \preceq y\right\} \in \tau$ because

$\left\{y \in X: x^{\prime} \preceq y\right\}=\left\{y \in X: x^{\prime} \preceq y \preceq b\right\} \cup\left\{y \in X: x^{\prime}<y\right\} \in \tau$

and thus (by definition of $D$ ) we have $x^{\prime} \sim z_{i}$ for some $z_{i} \in Z^{\prime}$. Furthermore there exists $z_{j} \in Z^{\prime}$ such that $x \preceq z_{j} \preceq b$ (by definition of $Z$ ), and therefore the set $V:=\left\{y \in X: z_{i} \preceq y<z_{j}\right\}$ satisfies $x^{\prime} \in V \subseteq B$ and $V=B \cap\left\{y \in X: y<z_{j}\right\} \in \tau ;$ consequently $V \in \mathcal{B}$.

CASE 3. Suppose now $B=\{y \in X: a \preceq y<b\}$. If $a<x^{\prime}<b$ then Case 1 applies to $B^{\prime}=\{y \in X: a<y<b\}$. We may therefore assume $a \sim x^{\prime}$. Thus it follows that $B=\left\{y \in X: x^{\prime} \preceq y<b\right\} \in \tau$, and therefore $x^{\prime} \sim z_{i}$ for some $z_{i} \in Z^{\prime}$ because $\left\{y \in X: x^{\prime} \preceq y\right\}=B \cup\left\{y \in X: x^{\prime}<y\right\} \in \tau$. The following cases arise:

(3.a) If there exists $x$ such that $x^{\prime}<x<b$ then there exists $z_{j} \in Z$ with $x^{\prime}<x \preceq z_{j} \preceq b$. Therefore $V:=\left\{y \in X: z_{i} \preceq y<z_{j}\right\} \in \tau(V=$ 
$\left.B \cap\left\{y \in X: y<z_{j}\right\}\right)$ and thus $V \in \mathcal{B}$. The open set $V$ satisfies the requirement.

(3.b) If there is no $x$ such that $x^{\prime}<x<b$ then $B=\left\{y \in X: x^{\prime} \sim y\right\} \in \tau$, and reasoning as in (2.a) we conclude that $B$ itself satisfies the requirement for $V$.

CASE 4. Finally, suppose $B=\{y \in X: a<y \preceq b\}$. This case is similar to Case 3 .

We now proceed to prove the necessity of the condition. Let us fix a countable base $\mathcal{B}$ of $\tau$.

In order to prove that < is perfectly separable we apply the argument used by Mehta for POTS, which proceeds as follows. Firstly, we check that the set $C$ of ordered pairs of equivalence classes $\left(\{a]_{\sim},[b]_{\sim}\right)$ such that $a<b$ and $\{y \in X: a<y<b\}=\emptyset$ is countable: we may associate to any pair $\left([a]_{\sim},[b]_{\sim}\right)$ of that type an open set $B_{b}$ of the countable base $\mathcal{B}$ of $\tau$, in such a way that the map $C \rightarrow \mathcal{B}$ thus defined is injective. Indeed, given $\left([a]_{\sim},[b]_{\sim}\right) \in C$ the set $\{y \in X: y<b\}$ is open and contains $a$, thus there exists an open set $B_{b} \in \mathcal{B}$ satisfying $a \in B_{b} \subseteq\{y \in X: y<b\}$. Now, given two different pairs $\left([a]_{\sim},[b]_{\sim}\right)$ and $\left(\left[a^{\prime}\right]_{\sim},\left[b^{\prime}\right]_{\sim}\right)$ in $C$, we may assume $a<b \preceq a^{\prime}<b^{\prime}$ (the other possible case is $a^{\prime}<b^{\prime} \preceq a<b$, and a similar argument would apply); from $a^{\prime} \in B_{b^{\prime}}, a^{\prime} \notin B_{b}$ we deduce that the open sets assigned to those pairs are different. Secondly, we define a countable set $Z$ in the following way. We form a countable dense set $D$ for $\tau$ by selecting an element from each member of the base $\mathcal{B}$, and we form another countable set $Z^{\prime}$ by selecting an element from each class $[a]_{\sim}$ such that $\left([a]_{\sim},[b]_{\sim}\right) \in C$ for some $[b]_{\sim}$. Define $Z=D \cup Z^{\prime}$. We show that $<$ is perfectly separable through this set: given $a, b \in X$ such that $a<b$, if $\{y \in X: a<y<b\} \neq \emptyset$ then there exists $d \in D \subseteq Z$ such that $d \in\{y \in X: a<y<b\} \in \tau$ because $D$ is dense; on the other hand, if $\{y \in X: a<y<b\}=\emptyset$ then $\left([a]_{\sim},[b]_{\sim}\right) \in C$ and by definition there exists $z \in Z^{\prime}$ such that $z \in[a]_{\sim}$, that is, $z \sim a$ and thus $a \preceq z<b$.

We now prove that the second property claimed holds too. Given an equivalence class $[x]_{\sim}$ that satisfies $\{z \in X: x \preceq z\} \in \tau$, there exists $B_{x} \in \mathcal{B}$ such that $x \in B_{x} \subseteq$ $\{z \in X: x \preceq z\}$. If $[x]_{\sim}$ and $[y]_{\sim}$ are two different classes of $T$ then the basic open sets $B_{x}$ and $B_{y}$ assigned in this way are different, since we may assume $x<y$ and thus $x \in B_{x}, x \notin B_{y}$. It follows that the set $T$ is countable.

In general, it is not true that a second countable topological space remains second countable if the topology is weakened. However, this property does hold in the class of the topologies $\tau$ on a space ordered by a preference $(X,<)$ such that $(X,<, \tau)$ is a GPO-space:

COROLlaRY 1. If $(X,<, \tau)$ is a GPO-space such that $\tau$ is second countable, $\tau^{\prime}$ is a topology such that $\tau^{\prime} \leqslant \tau$ and $\left(X,<, \tau^{\prime}\right)$ is a GPO-space, then $\tau^{\prime}$ is second 
countable (in particular, $\tau_{o r}(<)$ is second countable).

Proof: From the characterisation above, since $(X,<, \tau)$ is a GPO-space such that $\tau$ is second countable, it follows that $<$ is perfectly separable (a condition which depends on the order alone and therefore is satisfied by $\left(X,<, \tau^{\prime}\right)$ too) and $\left\{[x]_{\sim} \in X / \sim:\{y \in X: x \preceq y\} \in \tau\right.$ or $\left.\{y \in X: y \preceq x\} \in \tau\right\}$ is countable. This latter condition will also be satisfied by $\tau^{\prime}$ because $\tau^{\prime} \leqslant \tau$. It follows that $\tau^{\prime}$ is second countable.

We now consider the first countability axiom. This property will be characterised in GPO-spaces in terms of the following known concepts:

Definition 4: If $<$ is a preference on $X$, the subset $A$ of $X$ is cofinal in $X$ if $X=\{x \in X$ : there exists $a \in A$ with $x \preceq a\}$. We define dually coinitial subset in $X$.

Proposition 5. Let $(X,<, \tau)$ be a GPO-space. Then, $\tau$ is first countable if and only if for each $x \in X$, both statements (a) and (b) hold:

(a) either $\{y \in X: y \preceq x\} \in \tau$ or $\{y \in X: x<y\}$ contains a coinitial sequence; and

(b) either $\{y \in X: x \preceq y\} \in \tau$ or $\{y \in X: y<x\}$ contains a cofinal sequence.

Proof: We first prove the necessity of the condition. Suppose that $\tau$ is first countable, and fix $x \in X$; we shall show that statement (a) holds, and (b) would follow from a similar argument. We assume that $\{y \in X: y \preceq x\} \notin \tau$ (otherwise it is obvious that (a) is satisfied). Let $\mathcal{B}$ be a countable base of neighbourhoods of $x$ that satisfies the condition given by Proposition 2. We must prove that $\{y \in X: x<y\}$ contains a coinitial sequence, that is, a sequence of elements such that if $x<z$ then there exists an element $b$ in the sequence satisfying $b \preceq z$. Let us observe that, for any $x<z$, the set $\{y \in X: y<z\}$ is open and the following cases may arise:

- There exists $U=\{y \in X: a<y<b\} \quad(\{y \in X: a \preceq y<b\})$ an element from the base $\mathcal{B}$ such that $x \in U \subseteq\{y \in X: y<z\}$; then $x<b \preceq z$.

- There exists $U=\{y \in X: a<y \preceq b\} \quad(\{y \in X: a \preceq y \preceq b\})$ an element from the base $\mathcal{B}$ such that $x \in U \subseteq\{y \in X: y<z\}$. Then $b<z$, and if $b \sim x$ it follows that

$$
\{y \in X: y \preceq x\}=\{y \in X: a<y \preceq b\} \cup\{y \in X: y<b\}
$$

(respectively $\{y \in X: y \preceq x\}=\{y \in X: a \preceq y \preceq b\} \cup\{y \in X: y<b\}$ ) and therefore we have the contradiction $\{y \in X: y \preceq x\} \in \tau$; thus, from $x \preceq b$ we obtain $x<b$. 
We have therefore proved that the sequence formed by the right extremes of every open set of the base $\mathcal{B}$ (that is, the elements $b$ considered in any of the described cases) is coinitial in $\{y \in X: y<x\}$.

Let us see now the sufficiency of the condition. For any $x \in X$, we define a countable collection $\mathcal{B}_{\boldsymbol{x}}$ of neighbourhoods of $\boldsymbol{x}$ according to the following procedure. From statement (a), we define:

$$
A_{0}:=\{y \in X: y \preceq x\} \text { if }\{y \in X: y \preceq x\} \in \tau ; A_{0}:=\emptyset \text { otherwise }
$$

and

if $\{y \in X: x<y\}$ has a coinitial sequence $\left\{z_{n}\right\}_{n \in \mathcal{N}}$ then $A_{n}:=\left\{y \in X: y<z_{n}\right\}$ for all $n$; otherwise $A_{n}:=\emptyset$ for all $n$.

We define $B_{0}$ and $\left\{B_{n}\right\}_{n \in N}$ by a similar procedure. Obviously all these sets are neighbourhoods of $x$.

Let us define $\mathcal{B}_{x}:=\left\{A_{i} \cap B_{j}: i, j \in \mathcal{N}\right\}$. We show that $\mathcal{B}_{x}$ is a base of neighbourhoods of $x$.

If $U \in \tau$ and $x \in U$ then we can take a convex open set $V$ that satisfies $x \in V \subseteq U$. We now find a member $W$ of $\mathcal{B}_{x}$ that satisfies $x \in W \subseteq V$. To this end we define two neighbourhoods $A$ and $B$ of $x$, in the following way.

If $\{y \in X: y \preceq x\} \in \tau$ then we define $A=\{y \in X: y \preceq x\}$. If this is not the case: because $\{y \in X: x<y\}$ has a coinitial sequence $\left\{z_{n}\right\}_{n \in \mathcal{N}}$ by (a) and, on the other hand, there must exist $z \in V$ such that $x<z$ (otherwise $\{y \in X: y \preceq x\}=V \cup$ $\{y \in X: y<x\} \in \tau$, a contradiction), then there exists $n \in \mathcal{N}$ such that $x<z_{n} \preceq z$, and by convexity of $V$ it follows that $z_{n} \in V$; let us define $A:=\left\{y \in X: y<z_{n}\right\}$. Notice that such $A$ is one of the neighbourhoods of $x$ appearing in the definition of $\mathcal{B}_{x}$, and it satisfies $A \cap\{y \in X: x \preceq y\} \subseteq U$.

We define $B$ in a similar way, and thus it satisfies $B \cap\{y \in X: y \preceq x\} \subseteq U$.

Now the neighbourhood $W:=A \cap B$ of $x$ belongs to $\mathcal{B}_{x}$ by definition, and it satisfies $A \cap B \subseteq U$.

The following Corollary will permit us to extend first countability to certain finer topologies, a property which does not hold in general:

CoRollary 2. If $(X,<, \tau)$ is a GPO-space such that $\tau$ is first countable, $\tau^{\prime}$ is a topology such that $\tau \leqslant \tau^{\prime}$ and $\left(X,<, \tau^{\prime}\right)$ is a GPO-space, then $\tau^{\prime}$ is first countable. In particular, if $<$ is a preference on $X$ such that $\tau_{o r}(<)$ is first countable then all the topologies $\tau$ such that $(X,<, \tau)$ is a GPO-space are first countable.

PROOF: In order to prove that $\tau^{\prime}$ is first countable we shall check that the characterisation in Proposition 5 is satisfied. By symmetry, we only need show that the first 
statement holds. For each $x \in X$, since $(X,<, \tau)$ is a GPO-space such that $\tau$ is first countable, it follows that either $\{y \in X: y \preceq x\} \in \tau$ or $\{y \in X: x<y\}$ has a coinitial sequence; if the former holds it is obvious that $\{y \in X: y \preceq x\} \in \tau^{\prime}$, and the latter is a property that only depends on the order.

We point out that first countability may be characterised in POTS by adapting the proof of Proposition 5, in the following terms:

Proposition 6. Let $\left(X,<, \tau_{o r}(<)\right)$ be a POTS. Then, $\tau_{o r}(<)$ is first countable if and only if: for each $x \in X,\{y \in X: x<y\}$ contains a coinitial sequence, and $\{y \in X: y<x\}$ contains a cofinal sequence.

Proof: From Proposition 5, the sufficiency of the condition is obvious. As for the necessity, fix $x \in X$ and a countable base

$$
\mathcal{B}_{x}:=\left\{\left\{x \in X: a_{i}<x<b_{i}\right\}, i \in \mathcal{N}\right\}
$$

of neighbourhoods of $x$. Then we show that $\left\{b_{i}: i \in \mathcal{N}\right\} \cap\{y \in X: x<y\}$ (to exclude the possibility $\left.b_{i}=+\infty\right)$ is coinitial in $\{y \in X: x<y\}$, and a similar proof would hold for $\{y \in X: y<x\}$. Indeed, if $x<z$ then $\{y \in X: y<z\}$ is a neighbourhood of $x$ and thus there exists $i$ such that $x \in\left\{y \in X: a_{i}<y<b_{i}\right\} \subseteq\{y \in X: y<z\}$. Therefore $b_{i} \preceq z$ and the result follows.

\section{REFERENCES}

[1] J.C.R. Alcantud and J. M. Gutiérrez, Saturated identifications and topological properties of GPO-spaces, Centre for Discrete and Applicable Mathematics Research Report Series 96, November 1995 (London School of Economics, London).

[2] E. Cech, Topological spaces, Academia (Czechoslovak Acadademy of Science, Praga, 1966).

[3] G. Debreu, 'Representation of a preference ordering by a numerical function', in Decision Processes, (R.M. Thrall, C.H. Coombs and R.L. Davis, Editors) (Wiley, New York, 1954), pp. $159-165$.

[4] G. Debreu, 'Continuity properties of Paretian utility', Internat. Econom. Rev. 5 (1964), 285-293.

[5] M.J. Faber, Metrizability in generalized ordered spaces (Mathematical Centre Tracts 53, Amsterdam, 1974).

[6] P.C. Fishburn, Utility theory for decision making (Wiley, New York, 1970).

[7] R.W. Heath, D.J. Lutzer and P.L. Zenor, 'Monotonically normal spaces', Trans. Amer. Math. Soc. 178 (1973), 481-493.

[8] D.J. Lutzer, 'On generalized ordered spaces', Dissertationes Math. 89 (1971), 1-36.

[9] G. Mehta, 'Ordered topological spaces and the theorems of Debreu and Peleg', Indian J. Pure Appl. Math. 14 (1983), 1174-1182.

[10] E. Michael, 'The product of a normal space and a metric space need not be normal', Bull. Amer. Math. Soc. 69 (1963), 375-376. 
[11] E. Michael, 'Paracompactness and the Lindelöff property in finite and countable Cartesian products', Compositio Math 23 (1971), 199-214.

[12] A. Wilansky, Topology for analysis (Robert E. Krieger Publishing Co., Malabar, Fl., 1983).

Facultad de Economía y Empresa

Universidad de Salamanca

E 37008 Salamanca

Spain

e-mail: jcr@gugu.usal.es 